\title{
A Framework for the Construction of Self-replicating Tilings
}

\author{
María del Carmen Moure
}

Received: 4 December 2007 / Revised: 12 June 2009 / Accepted: 4 August 2009 /

Published online: 27 August 2009

(C) Springer Science+Business Media, LLC 2009

\begin{abstract}
Dekking (Adv. Math. 44:78-104, 1982; J. Comb. Theory Ser. A 32:315$320,1982)$ provided an important method to compute the boundaries of lattice reptiles as a 'recurrent set' on a free group of a finite alphabet. That is, those tilings are generated by lattice translations of a single tile, and there is an expanding linear map that carries tiles to unions of tiles. The boundary of the tile is identified with a sequence of words in the alphabet obtained from an expanding endomorphism (substitution) on the alphabet. In this paper, Dekking's construction is generalized to address tilings with more than one tile, and to have the elements of the tilings be generated by both translation and rotations. Examples that fall within the scope of our main result include self-replicating multi-tiles, self-replicating tiles for crystallographic tilings and aperiodic tilings.
\end{abstract}

\section{Introduction}

A lattice tiling of $\mathbb{R}^{2}$ is said to be a self-replicating tiling, or just rep-tiling, if all tiles are translates of each other by elements of a lattice, and there is an expanding linear map $A$ of the plane that maps each tile to a union of other tiles. These tiles have sophisticated properties. There are two methods to construct the tiles, and their properties. The first is to construct the tile from a iterated function system (IFS). In that case, the tile $Q$ is the attractor of an IFS with some properties, and satisfies the self-affine equation $Q=\bigcup s_{i}(Q)$, where $s_{i}$ are affine contractions.

The second, a subtle method initiated in the work of Dekking [2, 3], and subsequently refined by Vince [15], provided a framework for constructing the boundaries of rep-tiles, as a "recurrent set".

M.d.C. Moure ( $₫)$

Universidad Nacional de Mar del Plata, 7600 Mar del Plata, Argentina

e-mail:mcmoure@mdp.edu.ar 
In this paper, we continue the line of investigation of Dekking and Vince. An important element of our work is that we permit more than one tile in our tilings, that is, we study multi-tiles. We also permit rotations, so that our tilings are more general than lattice tilings. As is seen from our examples, our main theorem contains a wide repertoire of self-replicating tiles, including crystallographic multi-tiles, and aperiodic tilings.

We provide a new geometric representation of the cancellation that can occur in endomorphisms of free groups. The framework of Dekking [2, 3], works on the free semigroup generated by the alphabet $S$. We extend the formalism to work on the free group generated by $S$, providing a more elegant treatment of the cancellations.

Our extension of Dekking's formalism, once programmed, can be used to produce a large variety of fractal tilings. Moreover, we believe that it might be a useful tool to gain control of geometric properties of the fractal to be generated. For example, the endomorphism gives an upper estimate of the Hausdorff dimension for the recurrent set $[1,2]$ (see (5) on p. 725 and Sect. 3.3 at the end).

We show (Theorem 2(iv)) that the Hausdorff dimension being smaller than 2 guarantees that the self-replicating set tiles the plane by the action of the appropriate motions: translations, crystallographic groups or other ones.

For reference about papers on geometrical properties of fractal tiles, in particular related to the Hausdorff dimension of their boundaries, see, for example, [4, 11, 14].

In addition to the geometric appeal of fractal tiles, they have important connections to the construction of wavelets. A Haar type wavelet basis (multi-wavelet basis) can be constructed using the characteristic function(s) of a self-affine tile (multitile) $[5,7]$. Recently, more general systems have been studied, in which the basis elements contain not only translations and dilations of one (or finitely many) fixed functions, but one also allows some other type of motion [9]. Some of self-replicating tilings that we build from Theorem 2 can be used in this context [6].

The paper is organized as follows. In Sect. 2, we introduce some preliminary definitions and results. The problem around cancellations is depicted in this section. In Sect. 3, we will develop our framework. First, in Sect. 3.1, we study the simplest way, in which only translations are allowed. In Sect. 3.2, we introduce a more general framework which makes it possible to rotate the shapes and, in Sect. 3.3 an example is shown. This example also eases understanding of the following section. Finally, Sect. 4 contains our extension of Dekking's theorem [3] and some examples to illustrate our theorems.

\section{Background and Definitions}

We now recall the notation and basic results of [2, 3]. Let $S=\left\{s_{1}, s_{2}, \ldots, s_{r}\right\}$ be a finite alphabet, and $S^{*}$ the free semigroup generated by $S$, and $\theta: S^{*} \rightarrow S^{*}$ an endomorphism. Let $f: S^{*} \rightarrow \mathbb{R}^{d}$ be a homomorphism, that is, $f$ satisfies

$$
f(V W)=f(V)+f(W)
$$

for all words $V, W \in S^{*}$. The map $f$ is used to describe relative position. We need a way to associate compact subsets of $\mathbb{R}^{d}$ with words in $S^{*}$. Denote by $\mathcal{K}\left(\mathbb{R}^{d}\right)$ the 
space of compact subsets of $\mathbb{R}^{d}$. Any map $K[\cdot]: S \rightarrow \mathcal{K}\left(\mathbb{R}^{d}\right)$ is extended to nonempty words by requiring for all nonempty $V, W \in S^{*}$

$$
K[V W]=K[V] \cup(K[W]+f(V)) .
$$

(Notation: $A+y=\{x+y: x \in A\}$ )

An important example of such a map is the polygon map defined by (1) and for $s \in S$

$$
K[s]=\{t f(s): 0 \leq t \leq 1\} .
$$

If one considers endomorphisms of the free group $G$ generated by $S$ instead of the semigroup $S^{*}$, and $\theta$ and $f$ are extended in a natural way to $G$, then (1) will no longer make sense because of cancellations.

The way that Dekking proposes to adapt the definition of $K$ for free groups is to extend the map $K[\cdot]: S \cup S^{-1} \rightarrow \mathcal{K}\left(\mathbb{R}^{d}\right)$ to reduced words only. That is, for $W=$ $s_{1} s_{2} \cdots s_{n}$ a reduced word is

$$
K\left[s_{1} s_{2} \cdots s_{n}\right]=K\left[s_{1}\right] \cup\left(K\left[s_{2}\right]+f\left(s_{1}\right)\right) \cup \cdots \cup\left(K\left[s_{n}\right]+f\left(s_{1} \cdots s_{n-1}\right)\right),
$$

in this manner for $V, W$ reduced words we have

$$
K[V W] \subset K[V] \cup(K[W]+f(V)) .
$$

However, cancellations can occur not only in the word but also in the "run" of the associated set. For example, for a word $V W V^{-1}$ with $f(W)=0, K\left[V W V^{-1}\right]$ should be the translate of $K[W]$ to $f(V)$; however, it is not if we apply (3). This particular situation was pointed out and corrected in the paper of Ito [10, p. 303].

Our purpose is not to eliminate cancellations as in the already mentioned work of Ito. On the contrary, our aim is to give an appropriate representation of cancellations in the geometric realization of endomorphism of free groups, associating the alphabet of generators with line paths in an environment that allows a better handling of inverse elements and cancellations. To do that, we introduce a formalism that maps the words in the free group to a free Abelian group whose base is a family of segmented paths. In this manner, we capture the idea of cancellation even if it does not occur in the word but in the associated polygonal path. This will be very useful when, for example, a word represents the boundary of the union of tiles, and paths with opposite sign can represent the common part of the boundary of adjacent tiles.

Suppose now that $L_{\theta}: \mathbb{R}^{d} \rightarrow \mathbb{R}^{d}$ is a linear map such that

$$
f \theta(s)=L_{\theta} f(s)
$$

for all $s \in S$, where $f: S^{*} \rightarrow \mathbb{R}^{d}$ is the homomorphism specified above. We call $L_{\theta}$ a representation of $\theta$. To see existence conditions of $L_{\theta}$, see [2].

If all eigenvalues of an endomorphism $L$ of $\mathbb{R}^{d}$ have moduli larger than 1 , then we call $L$ expansive.

An endomorphism $\theta$ is called null-free if $\theta(s) \neq e$ (the empty word) for all $s \in S$. 
Theorem 1 (Dekking) Let $\theta$ be a null-free endomorphism of $S^{*}$, and $L_{\theta}$ an expansive representation of $\theta$. Then, for any nonempty word $W$, there exists a compact set $K_{\theta}(W)$ such that

$$
L_{\theta}^{-n} K\left[\theta^{n} W\right] \underset{n \rightarrow \infty}{\longrightarrow} K_{\theta}(W)
$$

in the Hausdorff metric. The set $K_{\theta}(W)$ does not depend on the choice of $K[\cdot]$, and is a curve.

Remark 1 The calculation of an upper bound for the Hausdorff dimension of $K_{\theta}(W)$ requires an estimate of the growth rate in the length of the word $\theta^{n} W$. We can define a non-negative matrix $A_{\theta}=\left(a_{i j}\right)$ in $\mathbb{Z}^{r \times r}$ where $r=|S|$ and $a_{i j}$ is defined as the number of $s_{j}$ or $s_{j}^{-1}$ in the word $\theta\left(s_{i}\right)$. Let $\lambda_{S}$ be the eigenvalue of $A_{\theta}$ with the greatest modulus. Since $A_{\theta}$ is non-negative, $\lambda_{S} \in \mathbb{R}$ and $\lambda_{S}>0$ because of Perron's theorem. If $L_{\theta}: \mathbb{R}^{d} \rightarrow \mathbb{R}^{d}$ has eigenvalues $\lambda_{1}, \ldots, \lambda_{d}$ where $\left|\lambda_{1}\right| \geq\left|\lambda_{2}\right| \geq \cdots \geq\left|\lambda_{d}\right|>1$, we have

$$
\operatorname{dim} K_{\theta}(W) \leq d-1+\frac{\log \lambda_{S}-\sum_{i=2}^{d} \log \left|\lambda_{i}\right|}{\left|\lambda_{1}\right|} .
$$

Now we recall some basic definitions, results and notations about chains. A chain (in $\mathbb{R}^{d}$ ) is an expression of the form

$$
n_{1} \gamma_{1}+n_{2} \gamma_{2}+\cdots+n_{m} \gamma_{m}
$$

where each $\gamma_{i}$ is a continuous non-constant path in $\mathbb{R}^{d}$ and each $n_{i}$ is an integer.

To make this precise, define a chain $\gamma$ in $\mathbb{R}^{d}$ to be a function that assigns to every non-constant path in $\mathbb{R}^{d}$ some integer, with the property that the function is zero for all but a finite number of paths. If $\gamma_{1}, \ldots, \gamma_{m}$ are the paths for which the value is not zero and $\gamma\left(\gamma_{i}\right)=n_{i}$, we write the chain as $\gamma=n_{1} \gamma_{i}+\cdots+n_{m} \gamma_{m}$. From this definition, it is clear how to add and subtract chains: one just adds or subtracts the corresponding values on each path. In this way, the chains form an Abelian group.

Let us denote the image of the path $x:[0,1] \rightarrow \mathbb{R}^{d}$ by $\operatorname{Supp}(x)$, and call it the support of $x$, i.e.,

$$
\operatorname{Supp}(x)=\{x(t): t \in[0,1]\}
$$

and for a chain $\gamma$

$$
\operatorname{Supp}(\gamma)=\bigcup_{\gamma(x) \neq 0} \operatorname{Supp}(x) .
$$

We give some technical definitions that will be useful when the chain represents the oriented boundary of a compact set.

We want to define the notion of a closed chain. This will mean that each point occurs as many times as an initial point as it does as a final point, counting multiplicities correctly. The precise definition is as follows:

Definition 1 For $\gamma=n_{1} \gamma_{1}+\cdots+n_{m} \gamma_{m}$, if $\gamma_{i}$ is a path from $P_{i}$ to $Q_{i}$, we define $\gamma$ to be a closed chain if for each point $T, \sum_{P_{i}=T} n_{i}=\sum_{Q_{i}=T} n_{i}$. 
It is easy to verify that the sum or difference of closed chains is closed.

For what follows, we consider $\mathbb{R}^{2}$. We shall define the total signed change in angle of a chain around a point $P$ that we will call the winding number around $P$. Let $\gamma:[a, b] \rightarrow \mathbb{R}^{2} \backslash\{P\}$ be a differentiable path. We write $\gamma(t)-P=(x(t), y(t))$ and $r(t)=\|\gamma(t)-P\|$, and let $\vartheta_{a}$ be such that $\gamma(a)-P=r(a)\left(\cos \vartheta_{a}, \sin \vartheta_{a}\right)$. Then the function $\vartheta(t)=\vartheta_{a}+\int_{a}^{t} \frac{-y(\tau) x^{\prime}(\tau)+x(\tau) y^{\prime}(\tau)}{r(\tau)^{2}} d \tau$ is differentiable and is the only continuous function that satisfies $\gamma(t)-P=r(t)(\cos \vartheta(t), \sin \vartheta(t))$ and $\vartheta(a)=\vartheta_{a}$.

Definition 2 For $P=\left(x_{0}, y_{0}\right)$ we denote by $\omega_{P}:=\frac{-\left(y-y_{0}\right) d x+\left(x-x_{0}\right) d y}{\left(x-x_{0}\right)^{2}+\left(y-y_{0}\right)^{2}}$ a 1 -form on $\mathbb{R}^{2} \backslash\{P\}$ and for a differentiable path $\gamma:[a, b] \rightarrow \mathbb{R}^{2} \backslash\{P\}$ we define the winding number of $\gamma$ around $P$ by

$$
\operatorname{Wind}(\gamma, P)=\frac{1}{2 \pi} \int_{\gamma} \omega_{P}=\frac{1}{2 \pi}(\vartheta(b)-\vartheta(a)),
$$

and then, for a chain $\gamma=\sum_{i=1}^{m} n_{i} \gamma_{i} ; P \notin \operatorname{Supp}(\gamma)$, the winding number will be

$$
\operatorname{Wind}(\gamma, P)=\sum_{i=1}^{m} n_{i}\left(\frac{1}{2 \pi} \int_{\gamma_{i}} \omega_{P}\right)=\sum_{i=1}^{m} n_{i} \operatorname{Wind}\left(\gamma_{i}, P\right) \text {. }
$$

Now, for a chain $\gamma=\sum_{i=1}^{m} n_{i} \gamma_{i}$ and $v \in \mathbb{R}^{2}$, we shall denote by $\gamma \overrightarrow{+} v$ the chain that results translating by $v$ each path $\gamma_{i}$, i.e., $\gamma \overrightarrow{+} v=\sum_{i=1}^{m} n_{i} \gamma_{i}^{\prime}$ where $\gamma_{i}^{\prime}(t)=$ $\gamma_{i}(t)+v$ (the usual addition in $\mathbb{R}^{2}$ ).

The following results can be proved:

(1) For $\gamma$ a path in $\mathbb{R}^{2} \backslash\{P\}$ and $v \in \mathbb{R}^{2}$, Wind $(\gamma \overrightarrow{+} v, P+v)=\operatorname{Wind}(\gamma, P)$.

(2) If $\gamma$ is a closed path and $P \notin \operatorname{Supp}(\gamma)$, then $\operatorname{Wind}(\gamma, P)$ is an integer.

(3) If $\gamma$ is a closed path and $P \notin \operatorname{Supp}(\gamma)$, Wind $(\gamma, P)$ is constant on each connected component of $\mathbb{R}^{2} \backslash \operatorname{Supp}(\gamma)$, as a function of $P$. It vanishes on the unbounded component.

\section{Chains Associated to Representations of Free Group Endomorphisms}

In order to simplify the presentation, we will split the explanation into two parts.

\subsection{Without Adding Directions}

Let $S, G, \theta, f$, and $L_{\theta}$ be as before. In addition, assume that, for $s_{i} \neq s_{j}$ in $S, f\left(s_{i}\right)$ and $f\left(s_{j}\right)$ are linearly independent. For each $s \in S$ and $v \in f(G)$, we consider the path $\gamma_{s v}$ defined by

$$
\gamma_{s v}(t)=t f(s)+v, \quad t \in[0,1] .
$$

We shall denote with $\mathcal{G}$ the family of all these line paths, i.e.,

$$
\mathcal{G}=\left\{\gamma_{s v}: s \in S, v \in f(G)\right\},
$$


and with $C(\mathcal{G})$ the group of chains on $\mathcal{G}$, i.e.,

$$
C(\mathcal{G})=\left\{\gamma: \gamma=n_{1} \gamma_{1}+\cdots+n_{m} \gamma_{m}, \gamma_{i} \in \mathcal{G}\right\}
$$

It is in this framework that we shall define the map $K[\cdot]: G \rightarrow \mathcal{K}\left(\mathbb{R}^{d}\right)$. For this, we just assign a chain to each word in $G$ as follows:

Definition 3 For the empty word $e$, define $k_{e}=0$.

For $V=s_{V 1}^{\alpha_{V 1}} s_{V 2}^{\alpha_{V 2}} \cdots s_{V m}^{\alpha_{V m}}$ with $s_{V i} \in S, \alpha_{V i}= \pm 1$, define $k_{V}:=\sum_{i=1}^{m} \alpha_{V i} \gamma_{i}$, where $\gamma_{i}$ is the segmental path (in $\mathcal{G}$ ) $\gamma_{s v}$ according to

$$
\begin{aligned}
& s=s_{V i}, v=f\left(s_{V 1}^{\alpha_{V 1}} s_{V 2}^{\alpha_{V 2}} \cdots s_{V i-1}^{\alpha_{V i-1}}\right) \quad \text { if } \alpha_{V i}=1, \\
& s=s_{V i}, v=f\left(s_{V 1}^{\alpha_{V 1}} s_{V 2}^{\alpha_{V 2}} \cdots s_{V i}^{\alpha_{V i}}\right) \quad \text { if } \alpha_{V i}=-1 .
\end{aligned}
$$

Thus, if $\alpha_{V i}=1, \gamma_{i}$ will be the line path from $f\left(s_{V 1}^{\alpha_{V 1}} s_{V 2}^{\alpha_{V 2}} \cdots s_{V i-1}^{\alpha_{V i-1}}\right)$ to $f\left(s_{V 1}^{\alpha_{V 1}} s_{V 2}^{\alpha_{V 2}} \cdots s_{V i}^{\alpha_{V i}}\right)$ and from $f\left(s_{V 1}^{\alpha_{V 1}} s_{V 2}^{\alpha_{V 2}} \cdots s_{V i}^{\alpha_{V i}}\right)$ to $f\left(s_{V 1}^{\alpha_{V 1}} s_{V 2}^{\alpha_{V 2}} \cdots s_{V i-1}^{\alpha_{V i-1}}\right)$ if $\alpha_{V i}=-1$.

Now, we define the map

$$
\begin{aligned}
& K[\cdot]: G \rightarrow \mathcal{K}\left(\mathbb{R}^{d}\right), \\
& K[V]=\operatorname{Supp}\left(k_{V}\right) .
\end{aligned}
$$

\section{Remark 2}

(i) For a reduced word $V$, some line segments may be canceled in $k_{V}$.

(ii) $K[V]$ may be disconnected.

Remark 3 It is not hard to see that if $K[V]$ (with $f(V)=0$ ) is the boundary of a polygon and $K[W]$ is the boundary of a compact set which can be tiled by $m$ translations of the polygon $K[V]$ using only translates from $f(G)$, then there are words $S_{1}, S_{2}, \ldots, S_{m}$ in $G$ such that

$$
W=S_{1} V S_{1}^{-1} S_{2} V S_{2}^{-1} \cdots S_{m} V S_{m}^{-1}
$$

This can be proved by induction on $m$.

The previous remark and Propositions 1 and 2 are the keys in our Theorem 2, once we add the directional ingredient of Sect. 3.2. Using propositions below, for $W=\theta V$ in (8) we can write

$$
\begin{aligned}
k_{V}^{(n)} & =L_{\theta}^{-n}\left(k_{\theta^{n} V}\right) \\
& =\left(k_{V}^{(n-1)} \overrightarrow{+} f\left(S_{1}\right)\right)+\left(k_{V}^{(n-1)} \overrightarrow{+} f\left(S_{2}\right)\right)+\cdots+\left(k_{V}^{(n-1)} \overrightarrow{+} f\left(S_{m}\right)\right) .
\end{aligned}
$$

The following proposition shows the basic properties of chains associated to words in $G$. 
Proposition 1 Let $V, W \in G$.

(i) $k_{V W}=k_{V}+\left(k_{W} \overrightarrow{+} f(V)\right)$, and in particular $k_{V^{-1}}=-\left(k_{V} \overrightarrow{+} f\left(V^{-1}\right)\right)=$ $-k_{V} \overrightarrow{+} f\left(V^{-1}\right)$.

(ii) If $f(V)=0$ then $k_{V}$ is a closed chain and $K[V]$ is a closed polygonal or a finite union of closed polygons.

(iii) If $V=A V^{\prime} A^{-1}$ with $A, V^{\prime}$ in $G$ and $f\left(V^{\prime}\right)=0$ then $k_{V}=k_{V^{\prime}} \overrightarrow{+} f(A)$ and $K[V]=K\left[V^{\prime}\right]+f(A)$.

(iv) If $k_{V}=k_{W}$ and $\theta$ is an endomorphism of $G$, then $k_{\theta V}=k_{\theta W}$.

Proof (i), (ii) and (iii) are easy to verify. To prove (iv), it will be sufficient to show that if $k_{V}=\sum_{i=1}^{l} n_{i} \gamma_{s_{i}} f\left(V_{i}\right)$ then $k_{\theta V}=\sum_{i=1}^{l} n_{i}\left(k_{\theta s_{i}} \overrightarrow{+} f\left(\theta V_{i}\right)\right)$, i.e., it does not depend on the word but on the chain. This can easily be seen by induction on the length of the word.

Remembering that our goal is to work on $L_{\theta}^{-n} K\left[\theta^{n} W\right]$, we define a homomorphism $\phi_{n}$ to go from a level $n-1$ to $n$. Let $\mathcal{G}_{0}=\mathcal{G}$, and for $n \in \mathbb{N}, \mathcal{G}_{n}=L_{\theta}^{-n}(\mathcal{G})$ be a new family of line paths, and let $C\left(\mathcal{G}_{n}\right)$ be the group of chains on $\mathcal{G}_{n}$ that we can identify with $L_{\theta}^{-n}(C(\mathcal{G}))$ by $\sum_{i=1}^{m} n_{i} L_{\theta}^{-1}\left(\gamma_{i}\right)$ with $L_{\theta}^{-1}\left(\sum_{i=1}^{m} n_{i} \gamma_{i}\right)$.

Definition 4 Let $\phi_{n}: C\left(\mathcal{G}_{n-1}\right) \rightarrow C\left(\mathcal{G}_{n}\right)$ be the homomorphism such that $\phi_{n}\left(L_{\theta}^{-(n-1)}\left(\gamma_{s v}\right)\right)=L_{\theta}^{-(n-1)}\left(L_{\theta}^{-1}\left(k_{\theta s}\right) \overrightarrow{+} v\right)$.

Recall that, by (4), $L_{\theta} f(G) \subset f(G)$, and therefore $L_{\theta}^{-(n-1)} f(G) \subset L_{\theta}^{-n} f(G)$.

We will denote $k_{V}^{(n)}=L_{\theta}^{-n}\left(k_{\theta^{n} V}\right)$.

Proposition 2 Let $V, W \in G$ and $n \in \mathbb{N}$.

(i) $k_{V W}^{(n)}=k_{V}^{(n)}+\left(k_{W}^{(n)} \overrightarrow{+} f(V)\right)$, and in particular $-k_{V}^{(n)}=k_{V^{-1}}^{(n)} \overrightarrow{+} f(V)$.

(ii) $\phi_{n}\left(k_{V}^{(n-1)}\right)=k_{V}^{(n)}$.

Proof From Proposition 1 and (4),

$$
k_{V W}^{(n)}=L_{\theta}^{-n}\left(k_{\theta^{n}(V W)}\right)=L_{\theta}^{-n}\left(k_{\theta^{n} V}+\left(k_{\theta^{n} W} \overrightarrow{+} f\left(\theta^{n} V\right)\right)\right)=k_{V}^{(n)}+\left(k_{W}^{(n)} \overrightarrow{+} f(V)\right) .
$$

In particular,

$$
-k_{V}^{(n)}=k_{V^{-1}}^{(n)} \overrightarrow{+} f(V) .
$$

Thus, for $s \in S$, if $\theta^{n-1}(s)=w_{1} w_{2} \cdots w_{l}$, we have

$$
\begin{aligned}
\phi_{n}\left(k_{s}^{(n-1)}\right)= & \phi_{n}\left(L_{\theta}^{-(n-1)}\left(k_{\theta^{n-1} s}\right)\right) \\
= & \phi_{n}\left(L_{\theta}^{-(n-1)}\left(k_{w_{1}}+\left(k_{w_{2}} \overrightarrow{+} f\left(w_{1}\right)\right)+\cdots+\left(k_{w_{l}} \overrightarrow{+} f\left(w_{1} \cdots w_{l-1}\right)\right)\right)\right) \\
= & L_{\theta}^{-(n-1)}\left(L_{\theta}^{-1} k_{\theta w_{1}}+\left(L_{\theta}^{-1} k_{\theta w_{2}} \overrightarrow{+} f\left(w_{1}\right)\right)+\cdots\right. \\
& \left.+\left(L_{\theta}^{-1} k_{\theta w_{l}} \overrightarrow{+} f\left(w_{1} \cdots w_{l-1}\right)\right)\right) \\
= & L_{\theta}^{-(n-1)}\left(L_{\theta}^{-1} k_{\theta w_{1}}+\left(L_{\theta}^{-1} k_{\theta w_{2}} \overrightarrow{+} L_{\theta}^{-1} f \theta\left(w_{1}\right)\right)+\cdots\right.
\end{aligned}
$$




$$
\begin{aligned}
& \left.\quad+\left(L_{\theta}^{-1} k_{\theta w_{l}} \overrightarrow{+} L_{\theta}^{-1} f \theta\left(w_{1} \cdots w_{l-1}\right)\right)\right) \\
& =L_{\theta}^{-n}\left(k_{\theta^{n} s}\right)=k_{s}^{(n)} .
\end{aligned}
$$

and, in a similar way, $\phi_{n}\left(k_{s}^{(n-1)} \overrightarrow{+} v\right)=k_{s}^{(n)} \overrightarrow{+} v$.

Now, by (i), it follows that if $V=z_{1} z_{2} \cdots z_{m} ; z_{i} \in S \cup S^{-1}$, then

$$
\begin{aligned}
\phi_{n}\left(k_{V}^{(n-1)}\right) & =\phi_{n}\left(k_{z_{1}}^{(n-1)}+\left(k_{z_{2}}^{(n-1)} \overrightarrow{+} f\left(z_{1}\right)\right)+\cdots+\left(k_{z_{m}}^{(n-1)} \overrightarrow{+} f\left(z_{1} z_{2} \cdots z_{m-1}\right)\right)\right) \\
& =k_{z_{1}}^{(n)}+\left(k_{z_{2}}^{(n)} \overrightarrow{+} f\left(z_{1}\right)\right)+\cdots+\left(k_{z_{m}}^{(n)} \overrightarrow{+} f\left(z_{1} z_{2} \cdots z_{m-1}\right)\right)=k_{V}^{(n)} .
\end{aligned}
$$

\subsection{Rotating Shapes and Adding Directions}

In what follows, we define the structure that will enable us to rotate the shapes, adding a directional component to the elements of the group.

Definition 5 Given a finite set $S=\left\{s_{1}, s_{2}, \ldots, s_{r}\right\}$, we will call $G$ the group generated by $X=\left\{(s, \varphi): s \in S \cup S^{-1}, \varphi \in \mathbb{R} / 2 \pi\right\}$, where the only relations in $G$ are $(s, \varphi)\left(s^{-1}, \varphi+\pi\right)=e$.

We will define an endomorphism $\theta$ of $G$ by means of assignment rules for each $s_{i} \in S$.

Choose $r$ natural numbers, $l_{1}, \ldots, l_{r}$. For each $i$, choose $l_{i}$ numbers $\left\{i_{1}, \ldots, i_{l_{i}}, 1 \leq\right.$ $\left.i_{j} \leq r\right\}, l_{i}$ signs $\left\{\alpha_{i_{1}}, \ldots, \alpha_{i_{l_{i}}}, \alpha_{i_{j}}= \pm 1\right\}$ and $l_{i}$ angles $\left\{\varphi_{i_{1}}, \ldots, \varphi_{i_{l_{i}}}, \varphi_{i_{j}} \in[0,2 \pi)\right\}$ and assign to $s_{i}$ the element $R_{i} \in G$ as follows:

$$
s_{i} \longrightarrow R_{i}=\left(s_{i_{1}}^{\alpha_{i_{1}}}, \varphi_{i_{1}}\right)\left(s_{i_{2}}^{\alpha_{i_{2}}}, \varphi_{i_{2}}\right) \cdots\left(s_{i_{l_{i}}}^{\alpha_{i_{l_{i}}}}, \varphi_{i_{l_{i}}}\right) \in G, \quad s_{i_{j}} \in S, \alpha_{i_{j}}= \pm 1 .
$$

Hence, $\theta: G \rightarrow G$ is defined by

$$
\theta\left(s_{i}, \xi\right)=\left(s_{i_{1}}^{\alpha_{i_{1}}}, \varphi_{i_{1}}+\xi\right)\left(s_{i_{2}}^{\alpha_{i_{2}}}, \varphi_{i_{2}}+\xi\right) \cdots\left(s_{i_{l_{i}}}^{\alpha_{l_{i}}}, \varphi_{i_{l_{i}}}+\xi\right)
$$

and since $\theta(s, \xi) \theta\left(s^{-1}, \xi+\pi\right)$ must be the empty word,

$$
\theta\left(s_{i}^{-1}, \xi\right)=\left(s_{i_{l_{i}}}^{-\alpha_{i_{i}}}, \varphi_{i_{l_{i}}}+\xi\right) \cdots\left(s_{i_{1}}^{-\alpha_{i_{1}}}, \varphi_{i_{1}}+\xi\right) .
$$

We want the homomorphism $f$ to change the direction of the vector according to the directional component $\varphi$ of the pair $(s, \varphi)$. For that, given a map $\mathbf{l}: S \rightarrow \mathbb{R}_{+}$such that $\mathbf{l}(s)=\mathbf{l}\left(s^{-1}\right)$, we define the homomorphism $f: G \rightarrow \mathbb{C}$ by

$$
f(s, \varphi)=\mathbf{l}(s) e^{i \varphi} .
$$

Note that $f(s, \varphi)^{-1}=f\left(s^{-1}, \varphi+\pi\right)=\mathbf{l}\left(s^{-1}\right) e^{i(\varphi+\pi)}=-\mathbf{l}(s) e^{i \varphi}=-f(s, \varphi)$.

We will call $L_{\theta}: \mathbb{R}^{2} \rightarrow \mathbb{R}^{2}$ (when it exists) the endomorphism such that $f \theta=L_{\theta} f$.

Finally, we define the polygonal lines associated to the elements of $G$. For that, we give a base for a group of chains as we have done in (6) replacing $S$ by $X$ and define the chains associated to the elements of $G$ as in Definition 3. 
Fig. 1 The figure shows a few rhombuses of the starting tiling. The small dotted triangle and lines are, respectively, the center of a rotation and the translation lines of a set of generators of the crystallographic group. Moving a pair of oriented edges by the group, we obtain the assignment of the edges

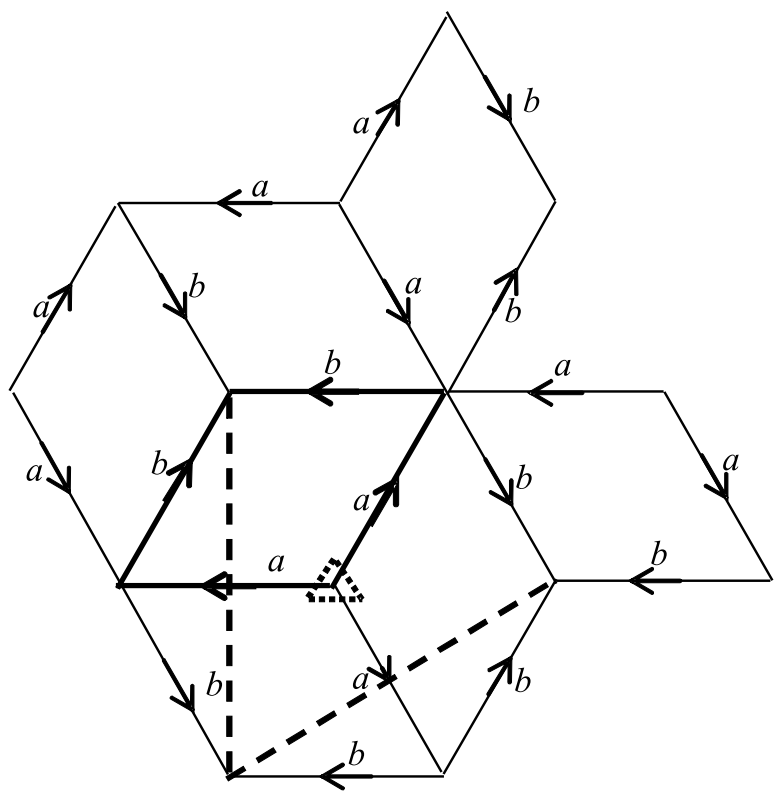

Note that the chain $k_{\left(s^{-1}, \varphi\right)}$ is the same as $k_{(s, \varphi)}$, but not $k_{\theta\left(s^{-1}, \varphi\right)}$ and $k_{\theta(s, \varphi)}$.

We can write for $V=\left(s_{1}, \varphi_{1}\right)\left(s_{2}, \varphi_{2}\right) \cdots\left(s_{k}, \varphi_{k}\right) \in G, s_{i} \in S \cup S^{-1}$

$$
\begin{aligned}
k_{V}= & k_{\left(s_{1}, \varphi_{1}\right)}+\left(k_{\left(s_{2}, \varphi_{2}\right)} \overrightarrow{+} f\left(s_{1}, \varphi_{1}\right)\right)+\cdots \\
& +\left(k_{\left(s_{k}, \varphi_{k}\right)} \overrightarrow{+}\left(f\left(s_{1}, \varphi_{1}\right)+\cdots+f\left(s_{k-1}, \varphi_{k-1}\right)\right)\right) .
\end{aligned}
$$

Now let $K[\cdot]: G \rightarrow \mathcal{K}\left(\mathbb{R}^{2}\right)$ be defined by $K[V]=\operatorname{Supp}\left(k_{V}\right)$.

To shorten the expressions, given an element $S=\left(s_{1}, \varphi_{1}\right)\left(s_{2}, \varphi_{2}\right) \cdots\left(s_{k}, \varphi_{k}\right)$ in $G$, we will denote $\xi S:=\left(s_{1}, \varphi_{1}+\xi\right)\left(s_{2}, \varphi_{2}+\xi\right) \cdots\left(s_{k}, \varphi_{k}+\xi\right)$.

With this, we are able to construct crystallographic tiles, as we will see in the next section. Before explaining the results and ideas about the generation of selfreplicating tiles, we turn to an example that may help to understand them.

\subsection{Crystallographic Tile Example}

We start with a tiling of the plane by rhombuses that tile the plane by the crystallographic group generated by a $\frac{2 \pi}{3}$ rotation and translations on a suitable lattice. We map the elements of the set $\left\{a, b, a^{-1}, b^{-1}\right\}$ to the four oriented edge of the rhombus, taking positive (counterclockwise) orientation in such a way that the common edge of two adjoining rhombuses, which necessarily have inverse direction, will get inverse assignments. In addition, we let the assignment be invariant by any similarity in the crystallographic group (that transforms the rhombus into another rhombus).

The element $\left(a, \frac{\pi}{3}\right)(b, \pi)\left(b^{-1}, 4 \frac{\pi}{3}\right)\left(a^{-1}, 0\right)$ determines the chain that is the positively oriented border of the rhombus chosen as initial figure (see Fig. 1). The rules 
Fig. 2 The figure shows the chain

$L_{\theta}^{-1} k_{\theta\left(a, \frac{\pi}{3}\right)(b, \pi)\left(b^{-1}, 4 \frac{\pi}{3}\right)\left(a^{-1}, 0\right)}$ over the initial rhombus. It is the positively oriented border of the union of 13 rhombuses that form a patch of the initial tiling transformed by the contraction $L_{\theta}^{-1}$

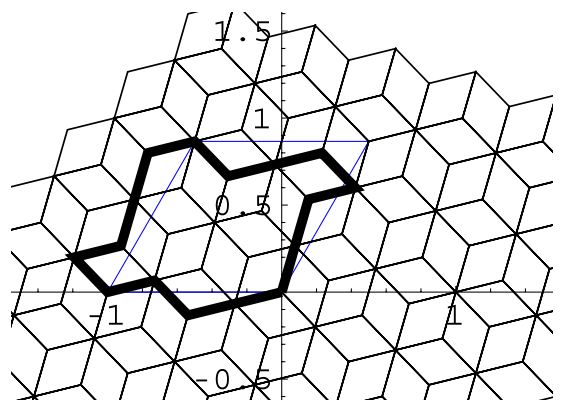

Fig. 3 Self-similar tile

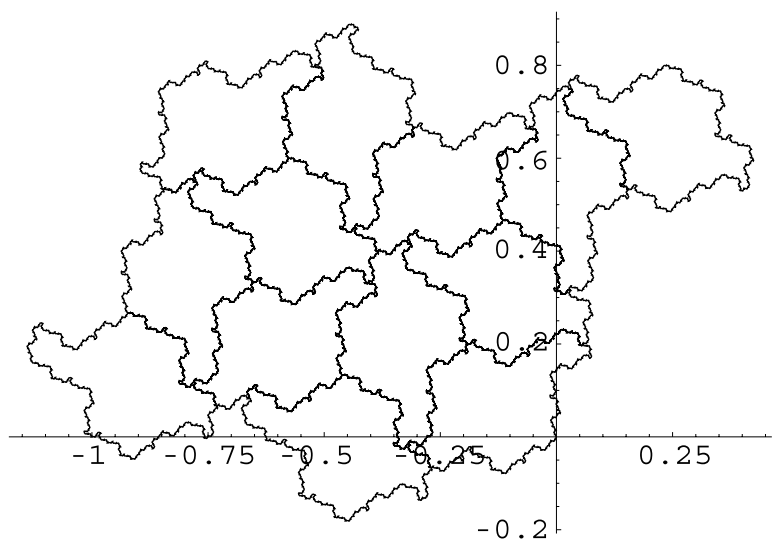

that define the endomorphism $\theta$ of $G$ (see (10) and (11)) are

$$
\begin{aligned}
& a \longrightarrow(a, 0)(b, 0)\left(b^{-1},-\frac{\pi}{3}\right)(b, 0) \\
& b \longrightarrow\left(b^{-1}, \frac{\pi}{3}\right)\left(a^{-1},-\frac{\pi}{3}\right)(a, 0)(b, 0)\left(b^{-1},-\frac{\pi}{3}\right) .
\end{aligned}
$$

If $\mathbf{I}(a)=\mathbf{l}(b)=1$, the homomorphism $f$ defined by (12) satisfies $f \theta=L_{\theta} f$ for $L_{\theta}(z)=e^{-i \omega} \sqrt{13} z$ where $\omega=\arctan (\sqrt{3} / 7)$.

The chain $L_{\theta}^{-1}\left(k_{\theta}\left(a, \frac{\pi}{3}\right)(b, \pi)\left(b^{-1}, 4 \frac{\pi}{3}\right)\left(a^{-1}, 0\right)\right.$ (see Fig. 2) is the positively oriented border of the union of 13 rhombuses that form a patch of the initial tiling transformed by the contraction $L_{\theta}^{-1}$, and each edge has the label obtained by applying $L_{\theta}^{-1}$ to the initial label. When tending to infinity, we obtain the self-similar figure that is shown in Fig. 3.

Now, we will calculate the upper bound given by Inequality (5). The non-negative matrix associated with $\theta$ is $A_{\theta}=\left(\begin{array}{ll}1 & 3 \\ 2 & 3\end{array}\right)$ whose largest eigenvalue is $2+\sqrt{7}$. Thus, $\operatorname{dim} K_{\theta} \leq \frac{2+\sqrt{7}}{\sqrt{13}}<2$. Then $K_{\theta}$ is not a space filling curve and Theorem 2(iv) says that the figure enclosed by $K_{\theta}$ tiles the plane under the action of the same crystallographic group as the initial rhombus. 


\section{Generating Boundaries of Self-affine Tiles and Multi-self-affine Tiles}

In [3], a theory for the construction of boundaries of self replicating plane sets is presented. We describe it here. For an endomorphism $\theta$ of a free group $G$ on two generators $a$ and $b$ and a homomorphism $f: G \rightarrow \mathbb{R}^{2}$, if the matrix of $L_{\theta}$ with respect to the basis $\{f(a), f(b)\}$ is $\lambda M$ where $|\lambda|>1$ with $M$ an orthogonal matrix and $K\left[\theta\left(a b a^{-1} b^{-1}\right)\right]$ is double-point free, then $L_{\theta}^{-n} K\left[\theta^{n}\left(a b a^{-1} b^{-1}\right)\right] \longrightarrow_{n \rightarrow \infty} K_{\theta}$ in the Hausdorff metric, where $K_{\theta}$ is the boundary of a $k$-replicating compact set $\left(k=\left|\operatorname{det} L_{\theta}\right|\right)$.

The idea of the method is that the set enclosed by $L_{\theta}^{-1} K\left[\theta\left(a b a^{-1} b^{-1}\right)\right]$ is tiled by $k$ parallelograms each of which is congruent to $L_{\theta}^{-1} K\left[a b a^{-1} b^{-1}\right]$. Further, $L_{\theta}^{-n} K\left[\theta^{n}\left(a b a^{-1} b^{-1}\right)\right]$ is tiled by $k$ copies of $L_{\theta}^{-n} K\left[\theta^{n-1}\left(a b a^{-1} b^{-1}\right)\right]$. Taking limits we have the result.

In [15], Vince shows that any self-replicating lattice tiling of the plane can be constructed using this method. But for many of them a large number of cancellations occur in $\theta^{n}\left(a b a^{-1} b^{-1}\right)$, which, in turn, makes it difficult to calculate other properties of the tiles, such as its Hausdorff dimension. Using polygons, not only parallelograms, we will have more possibilities to obtain better approximating polygons and to eliminate the cancellations. Furthermore, combining at least two polygons we can obtain multi-self-affine tiles, and finally, if it is possible to rotate the shapes, we can get multi-self-similar tiles that produce crystallographic tilings or even aperiodic tilings.

If $k_{W}$ is a closed chain such that $\left\{\operatorname{Wind}\left(k_{W}, x\right): x \notin K[W]=\operatorname{Supp}\left(k_{W}\right)\right\}=\{0,1\}$ or $\left\{\operatorname{Wind}\left(k_{W}, x\right): x \notin K[W]=\operatorname{Supp}\left(k_{W}\right)\right\}=\{0,-1\}$, we may consider $K[W]$ as the boundary of a compact (not necessarily connected) set and we define $\tilde{K}[W]$ as the closure of $\left\{x \in \mathbb{R}^{2}: \operatorname{Wind}\left(k_{W}, x\right) \neq 0\right\}$.

In most of the tilings $\mathcal{T} \ddot{=}\left\{T_{1}, T_{2}, \ldots\right\}$ the intersection of any finite set of tiles may be empty or may consist of a set of isolated points and arcs. In these cases, the points are called vertices of the tiling and the arcs will be called edges of the tiling.

In a tiling in which each tile is a polygon, if the vertices and edges of the polygons coincide with the vertices and edges of the tiling, we say that the tiling is edge-to-edge for more details see [9].

In the following theorem, we refer to a patch of tiles in a given tiling. By this we mean a finite number of tiles of the tiling with the property that their union is connected and simply connected.

We will say that a family of sets $\left\{S_{1}, S_{2}, \ldots, S_{k}\right\}$ are almost disjoint if the measure of $S_{i} \cap S_{j}$ is zero when $i \neq j$.

The theorem that we present here is similar to Theorem B in [12], but here we use the preceding framework which gives other possibilities of looking at the generation and production of examples. Moreover, under some conditions, the endomorphism $\theta$ allows us to calculate the Hausdorff dimension of the boundaries of the reptiles obtained [13].

We state our theorem for two sets $T_{1}$ and $T_{2}$ to make the theorem as clear as possible, but we note that the theorem holds with obvious modifications for a finite family of sets, or even for a single one. 
In what follows, we will assume given a group $G$ defined as in Definition 5, an endomorphism $\theta$ of $G$ which is given from assignment rules as (10) and (11) and a homomorphism $f$ defined by (12). Also assume that there is a map $L_{\theta}$ such that $f \theta=L_{\theta} f$, and $L_{\theta}$ is a rotation followed by a scalar multiplication and:

(A) $W_{1}$ and $W_{2}$ in $G$ are such that $k_{W_{1}}$ and $k_{W_{2}}$ are the boundary with positive orientation of two polygons $T_{1}$ and $T_{2}$ that tile the plane in an edge-toedge tiling $\mathcal{T}=\left\{\psi_{i}^{1}\left(T_{1}\right), \psi_{j}^{2}\left(T_{2}\right): i, j=1,2, \ldots\right\}$ where $\psi_{j}^{k}(z)=e^{i \varphi_{j}^{k}} z+v_{j}^{k}$. Moreover, if $\psi_{i}^{p}\left(T_{p}\right)$ and $\psi_{j}^{q}\left(T_{q}\right)$ have a common edge, this is canceled in $\left(k_{\varphi_{i}^{p} W_{p}} \overrightarrow{+} v_{i}^{p}\right)+\left(k_{\varphi_{j}^{q} W_{q}} \overrightarrow{+} v_{j}^{q}\right)$.

(B) $k_{\theta W_{1}}$ and $k_{\theta W_{2}}$ are the boundary with positive orientation of patches $\mathcal{P}_{1}$ and $\mathcal{P}_{2}$ of the tiling (or even a finite number of patches) such that the measure of $\mathcal{P}_{i}$ is $\left|\operatorname{det} L_{\theta}\right|$ times the measure of $T_{i}$ and if

$$
\mathcal{P}_{j}=\bigcup_{\kappa \in \mathcal{N}_{1}^{j}}\left(e^{i \varphi_{\kappa}^{1}}\left(T_{1}\right)+v_{\kappa}^{1}\right) \cup \bigcup_{\kappa \in \mathcal{N}_{2}^{j}}\left(e^{i \varphi_{\kappa}^{2}}\left(T_{2}\right)+v_{\kappa}^{2}\right),
$$

then

$$
k_{\theta W_{j}}=\sum_{\kappa \in \mathcal{N}_{1}^{j}}\left(k_{\varphi_{\kappa}^{1} W_{1}} \overrightarrow{+} v_{\kappa}^{1}\right)+\sum_{\kappa \in \mathcal{N}_{2}^{j}}\left(k_{\varphi_{\kappa}^{2} W_{2}} \overrightarrow{+} v_{\kappa}^{2}\right) .
$$

Actually, (14) could be obtained from (13) following Remark 3.

Theorem 2 Under hypotheses (A) and (B) above, if we define

$$
T_{j}^{n}:=L_{\theta}^{-1}\left(\bigcup_{\kappa \in \mathcal{N}_{1}^{j}}\left(e^{i \varphi_{\kappa}^{1}}\left(T_{1}^{n-1}\right)+v_{\kappa}^{1}\right) \cup \bigcup_{\kappa \in \mathcal{N}_{2}^{j}}\left(e^{i \varphi_{\kappa}^{2}}\left(T_{2}^{n-1}\right)+v_{\kappa}^{2}\right)\right),
$$

where $T_{j}^{0}=T_{j}$, then

(i) $L_{\theta}^{-n}\left(K\left[\theta^{n} W_{i}\right]\right)=\partial T_{i}^{n}$ and the sets in the union (15) are almost disjoint.

(ii) $\left\{\psi_{i}^{1}\left(T_{1}^{n}\right), \psi_{j}^{2}\left(T_{2}^{n}\right): i, j=1,2, \ldots\right\}$ is a tiling for each $n$.

(iii) If $\left(T_{1}^{*}, T_{2}^{*}\right)=\lim _{n \rightarrow \infty}\left(T_{1}^{n}, T_{2}^{n}\right)$ are the compact sets such that

$$
T_{j}^{*}:=L_{\theta}^{-1}\left(\bigcup_{\kappa \in \mathcal{N}_{1}^{j}}\left(e^{i \varphi_{\kappa}^{1}}\left(T_{1}^{*}\right)+v_{\kappa}^{1}\right) \cup \bigcup_{\kappa \in \mathcal{N}_{2}^{j}}\left(e^{i \varphi_{\kappa}^{2}}\left(T_{2}^{*}\right)+v_{\kappa}^{2}\right)\right),
$$

then the sets in the union in (16) are almost disjoint for both $j=1,2$, and $\left\{\psi_{i}^{1}\left(T_{1}^{*}\right), \psi_{j}^{2}\left(T_{2}^{*}\right): i, j=1,2, \ldots\right\}$ is a covering of $\mathbb{R}^{2}$.

(iv) If $K_{i}:=\lim _{n \rightarrow \infty} L_{\theta}^{-n}\left(K\left[\theta^{n} W_{i}\right]\right)$ are not space filling then $K_{i}=\partial T_{i}^{*}$ and $\left\{\psi_{i}^{1}\left(T_{1}^{*}\right), \psi_{j}^{2}\left(T_{2}^{*}\right): i, j=1,2, \ldots\right\}$ is a tiling.

Outline of the proof: We use (ii') to indicate a claim that includes (ii) and says: 
(ii') $\left\{\psi_{i}^{1}\left(T_{1}^{n}\right), \psi_{j}^{2}\left(T_{2}^{n}\right): i, j=1,2, \ldots\right\}$ is a tiling and the common edges of $\partial\left(\psi_{i}^{p}\left(T_{p}^{n}\right)\right)$ are canceled in the sum of the chains $k_{\varphi_{i}^{p} W_{p}}^{(n)} \overrightarrow{+} v_{i}^{p}$.

The claim (i) for $n=1$ follows from (A) and (B).

For (ii') with $n=1$, let $B\left(x_{0}, R\right)$ be a disk and

$$
\delta=\sup \left\{\sup \left\{\|x-y\|: x \in \operatorname{Supp}(k(s, \varphi)), y \in \operatorname{Supp}\left(k_{(s, \varphi)}^{(1)}\right)\right\} s \in S\right\} .
$$

Note that $\delta$ does not depend on $\varphi$.

Since $\left\{\psi_{i}^{1}\left(T_{1}\right), \psi_{j}^{2}\left(T_{2}\right): i, j=1,2, \ldots\right\}$ is a tiling, we can choose finite minimal sets $\mathcal{A}_{1}, \mathcal{A}_{2} \subset \mathbb{N}$ such that

$$
B\left(x_{0}, R+\delta\right) \subset \bigcup_{i \in \mathcal{A}_{1}} \psi_{i}^{1}\left(T_{1}\right) \cup \bigcup_{j \in \mathcal{A}_{2}} \psi_{j}^{2}\left(T_{2}\right) .
$$

Now, if we write

$$
\sum_{i \in \mathcal{A}_{1}}\left(k_{\varphi_{i}^{1} W_{1}} \overrightarrow{+} v_{i}^{1}\right)+\sum_{j \in \mathcal{A}_{2}}\left(k_{\varphi_{j}^{2} W_{2}} \overrightarrow{+} v_{j}^{2}\right)=\sum_{i=1}^{q}\left(k\left(s_{j_{i}}, \xi_{i}\right) \overrightarrow{+} v_{i}\right),
$$

it is not hard to see that due to cancellations

$$
\operatorname{Supp}\left(k\left(s_{j_{i}}, \xi_{i}\right) \overrightarrow{+} v_{i}\right) \cap B\left(x_{0}, R+\delta\right)=\emptyset \quad \text { for all } i=1,2, \ldots, q .
$$

Applying $\phi_{1}$ to (17), we obtain

$$
\sum_{i \in \mathcal{A}_{1}}\left(k_{\varphi_{i}^{1} W_{1}}^{(1)} \overrightarrow{+} v_{i}^{1}\right)+\sum_{j \in \mathcal{A}_{2}}\left(k_{\varphi_{j}^{2} W_{2}}^{(1)} \overrightarrow{+} v_{j}^{2}\right)=\sum_{i=1}^{q}\left(k^{(1)}\left(s_{j_{i}}, \xi_{i}\right) \overrightarrow{+} v_{i}\right),
$$

and for each $i=1,2, \ldots, q$

$$
\operatorname{dist}\left(\operatorname{Supp}\left(\left(k\left(s_{j_{i}}, \xi_{i}\right) \overrightarrow{+} v_{i}\right), \operatorname{Supp}\left(k^{(1)}\left(s_{j_{i}}, \xi_{i}\right) \overrightarrow{+} v_{i}\right)\right)\right) \leq \delta .
$$

Therefore,

$$
\operatorname{Supp}\left(k^{(1)}\left(s_{j_{i}}, \xi_{i}\right) \overrightarrow{+} v_{i}\right) \cap B\left(x_{0}, R\right)=\emptyset \quad \text { for all } i=1,2, \ldots, q,
$$

from which we have

$$
\operatorname{Wind}\left(\sum_{i \in \mathcal{A}_{1}}\left(k_{\varphi_{i}^{1} W_{1}}^{(1)} \overrightarrow{+} v_{i}^{1}\right)+\sum_{j \in \mathcal{A}_{2}}\left(k_{\varphi_{j}^{2} W_{2}}^{(1)} \overrightarrow{+} v_{j}^{2}\right), P\right) \equiv N \quad \text { for } P \text { in } B\left(x_{0}, R\right) \text {. }
$$

From (B) and (i) for $n=1$, it follows that

$$
\begin{aligned}
& \mathrm{m}\left(T_{1}\right)=\mathrm{m}\left(T_{1}^{1}\right)=\left|\operatorname{det} L_{\theta}\right|^{-1}\left(\#\left(\mathcal{N}_{1}^{1}\right) \mathrm{m}\left(T_{1}\right)+\#\left(\mathcal{N}_{2}^{1}\right) \mathrm{m}\left(T_{2}\right)\right), \\
& \mathrm{m}\left(T_{2}\right)=\mathrm{m}\left(T_{2}^{1}\right)=\left|\operatorname{det} L_{\theta}\right|^{-1}\left(\#\left(\mathcal{N}_{1}^{2}\right) \mathrm{m}\left(T_{1}\right)+\#\left(\mathcal{N}_{2}^{2}\right) \mathrm{m}\left(T_{2}\right)\right) .
\end{aligned}
$$


By the minimality of $\mathcal{A}_{1}$ and $\mathcal{A}_{2}$, it can be proved that, for $R$ sufficiently large, it must be true that $N=1$. Thus we have proved that (ii') is true for $n=1$.

Inductively, we can prove that if (i) and (ii') are true for $n$ then (i) is true for $n+1$, and (ii') being valid for $n$ and (i) for $n+1$ implies (ii') is true for $n+1$.

For (iii), it is easy to show that $\left\{\psi_{i}^{1}\left(T_{1}^{*}\right), \psi_{j}^{2}\left(T_{2}^{*}\right): i, j=1,2, \ldots\right\}$ is a covering of $\mathbb{R}^{2}$.

From Baire's category theorem, $T_{p}^{*}$ has a nonempty interior either for $p=1$ or $p=2$, and therefore for both $p=1$ and $p=2$. Moreover, if $\lambda=\mathrm{m}\left(T_{2}\right) / \mathrm{m}\left(T_{1}\right)$, we have

$$
\begin{aligned}
& \left|\operatorname{det} L_{\theta}\right|=\#\left(\mathcal{N}_{1}^{1}\right)+\#\left(\mathcal{N}_{2}^{1}\right) \lambda, \quad \text { and } \\
& \left|\operatorname{det} L_{\theta}\right|=\#\left(\mathcal{N}_{1}^{2}\right) \lambda^{-1}+\#\left(\mathcal{N}_{2}^{2}\right),
\end{aligned}
$$

which implies that the union (16) is essentially disjoint.

Remark 4 If $\varphi_{j}^{k}=0$ for all $k, j$, i.e., if the tiling is by translations, then $L_{\theta}$ can be affine.

Finally, (iv) can be proved as in [15, Theorem 3].

\subsection{Aperiodic Example}

Here $\mathcal{T}=\left\{\psi_{i}^{1}\left(T_{1}\right), \psi_{j}^{2}\left(T_{2}\right): i, j=1,2, \ldots\right\}$ is a Penrose aperiodic tiling by kites $\left(T_{1}\right)$ and darts $\left(T_{2}\right)$.

For $S=\{a, b\}$, if $\varphi=\pi / 5, W_{1}=(a,-\varphi)(b, 2 \varphi)\left(b^{-1}, 3 \varphi\right)\left(a^{-1}, 6 \varphi\right), W_{2}=$ $(b, 7 \varphi)(a, \varphi)\left(a^{-1}, 4 \varphi\right)\left(b^{-1}, 8 \varphi\right)$, and $\mathbf{I}(a)=\tau=\frac{1+\sqrt{5}}{2}, \mathbf{I}(b)=1$, then the chains $k_{W_{1}}$ and $k_{W_{2}}$ are the boundary with positive orientation of a pair of basic shapes $T_{1}$ and $T_{2}$. The position of inverse elements $s, s^{-1}$ indicates the way kites and darts are arranged in the Penrose tiling according to the coloring vertices restriction.

The rules

$$
\begin{aligned}
& a \longrightarrow(a,-3 \varphi)(b, 0)(a, 0)(b, \varphi)(a, \varphi), \\
& b \longrightarrow\left(a^{-1}, \varphi\right)\left(b^{-1}, \varphi\right)\left(a^{-1},-2 \varphi\right)
\end{aligned}
$$

define an endomorphism $\theta$ that satisfies $f \theta=L_{\theta} f$ for $L_{\theta}(z)=\tau^{2} z$. Moreover, $k_{\theta W_{1}}$ and $k_{\theta W_{2}}$ are the boundary with positive orientation of patches $\mathcal{P}_{1}$ and $\mathcal{P}_{2}$ (see Fig. 4)

Fig. 4 The shapes enclosed by $L_{\theta}^{-1}\left(k_{\theta W_{1}}\right)$ and $L_{\theta}^{-1}\left(k_{\theta W_{2}}\right)$
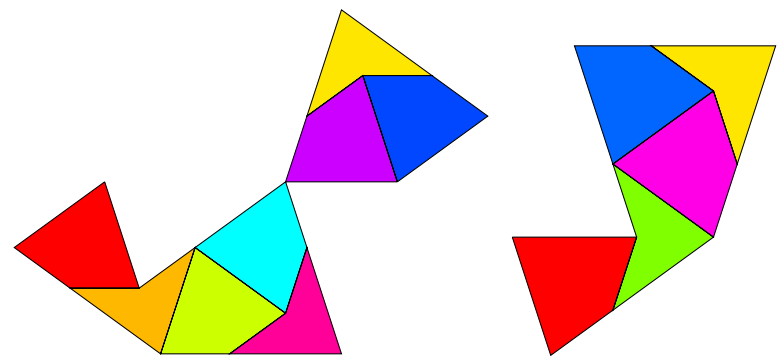
Fig. 5 Penrose self-similar tiles

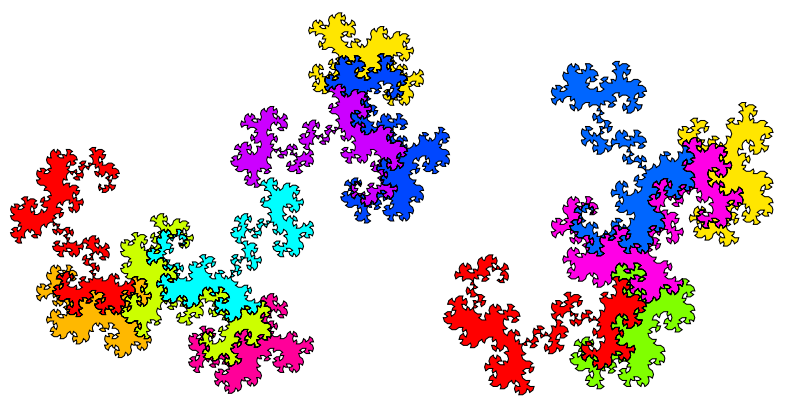

Fig. 6 Tiling

$\left\{\gamma\left(T_{1}\right), \gamma\left(T_{2}\right): \gamma \in \Gamma\right\}$

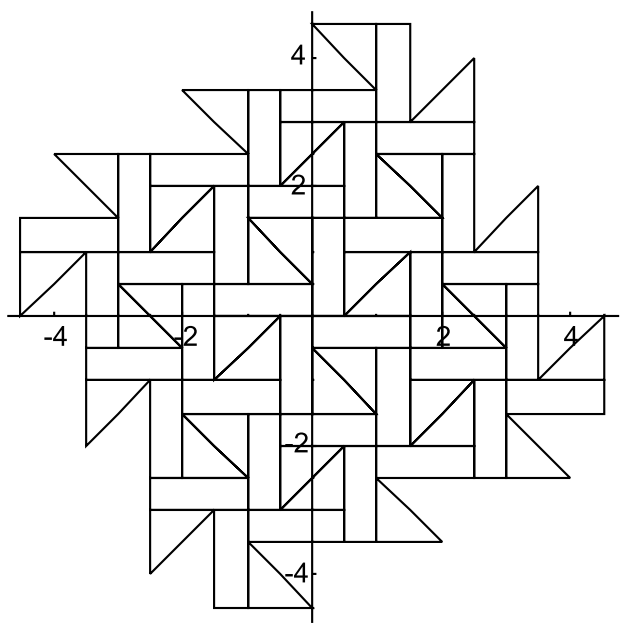

of the tiling $\mathcal{T}$ such that the measure of $\mathcal{P}_{i}$ is $\left|\operatorname{det} L_{\theta}\right|$ times the measure of $T_{i}$. Thus, the hypothesis (A) and (B) are satisfied. Figure 5 shows a good aproximation of the aperiodic self-similar tiles.

\subsection{Crystallographic Example with Two Tiles}

Let $\Gamma$ be the $p 4$ crystallographic group generated by $\left\langle R_{\pi / 2}, t_{(2,1)}, t_{(-1,2)}\right\rangle$, where $R_{\pi / 2}$ is the rotation around the origin and angle $\pi / 2, t_{(2,1)}$ and $t_{(-1,2)}$ are the translations in $(2,1)$ and $(-1,2)$, respectively. We start with a tiling by two polygons, a rectangle $T_{1}$ and a triangle $T 2$, such that $\left\{\gamma\left(T_{1}\right), \gamma\left(T_{2}\right): \gamma \in \Gamma\right\}$ is a tiling of the plane (see Fig. 6).

For $S=\{a, b, c, d, e\}$, if

$$
\begin{aligned}
& W_{1}=\left(c^{-1},-\pi / 2\right)(d, 0)\left(a^{-1}, 0\right)(a, \pi / 2)(b, \pi)(c, \pi), \\
& W_{2}=\left(c^{-1}, 0\right)\left(b^{-1}, 0\right)\left(d^{-1}, \pi / 2\right)(e, 5 \pi / 4)\left(e^{-1}, 5 \pi / 4\right)(c, \pi)
\end{aligned}
$$

and $\mathbf{l}(a)=\mathbf{l}(c)=\frac{1}{2}, \mathbf{l}(b)=\mathbf{l}(d)=1, \mathbf{l}(e)=\frac{\sqrt{2}}{2}$, then the chains $k_{W_{1}}$ and $k_{W_{2}}$ (Fig. 7) are the boundary with positive orientation of the pair of basic shapes $T_{1}$ and $T_{2}$. 
Fig. $7 k_{W_{1}}$ (rectangle) and $k_{W_{2}}$ (triangle)

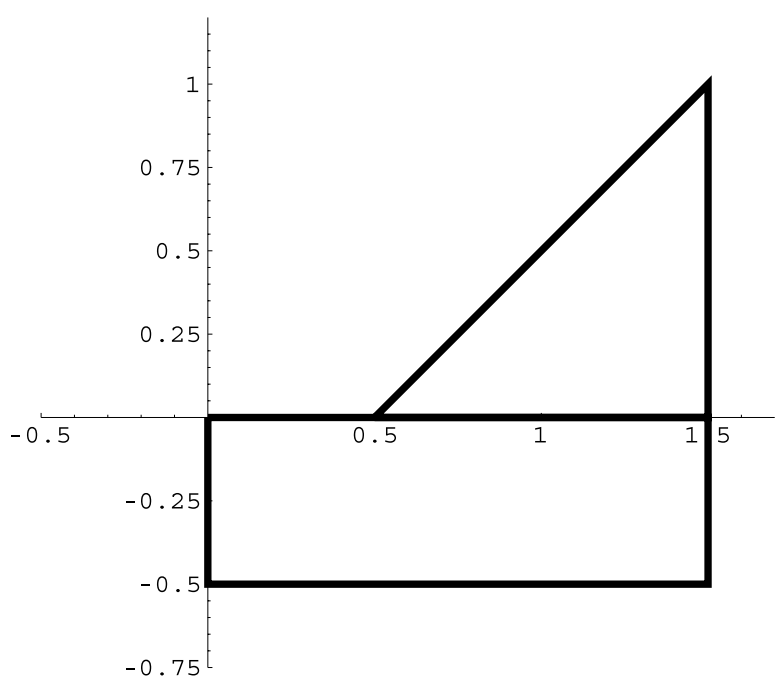

Fig. $8 L_{\theta}^{-1}\left(k_{\theta W_{1}}\right)$ and $L_{\theta}^{-1}\left(k_{\theta W_{2}}\right)$

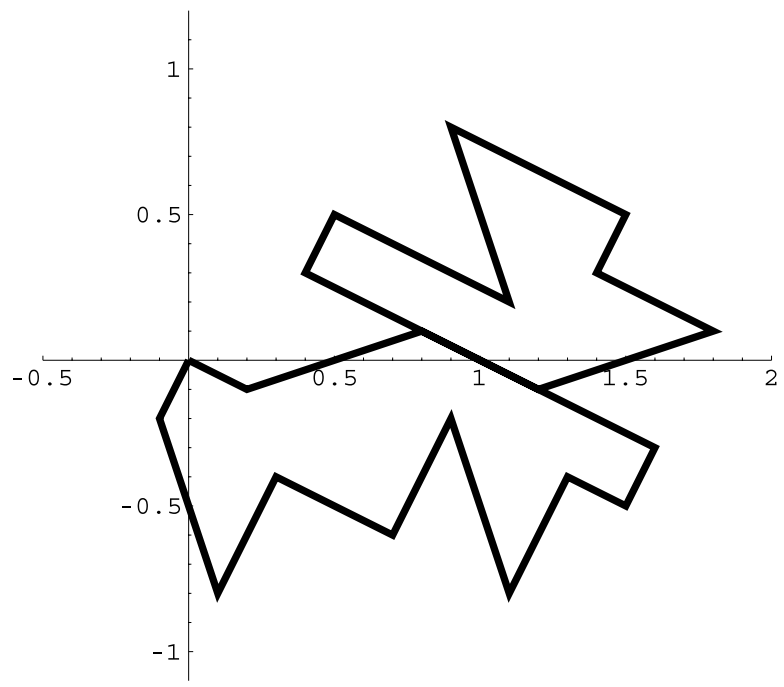

The rules defining the endomorphism are lengthy, and so omitted. Figures 8 and 9 show the first and fifth steps of the iteration respectively.

The expansive map $L_{\theta}=\sqrt{5} R_{\varphi}$ where $\varphi=\arctan \left(\frac{1}{2}\right) \in[0, \pi]$ satisfies $L_{\theta} \Gamma L_{\theta}^{-1} \subset$ $\Gamma$ and $f \theta=L_{\theta} f$.

We obtain two self-replicating sets

$$
T_{1}^{*}=L_{\theta}^{-1}\left(\left(\bigcup_{i=1}^{3} \gamma_{1, i}^{1}\left(T_{1}^{*}\right)\right) \cup\left(\bigcup_{i=1}^{3} \gamma_{1, i}^{2}\left(T_{2}^{*}\right)\right)\right),
$$


Fig. $9 L_{\theta}^{-5}\left(k_{\theta} W_{1}\right)$ and $L_{\theta}^{-5}\left(k_{\theta^{5} W_{2}}\right)$

Fig. $10 T_{1}^{*}$

Fig. $11 T_{2}^{*}$

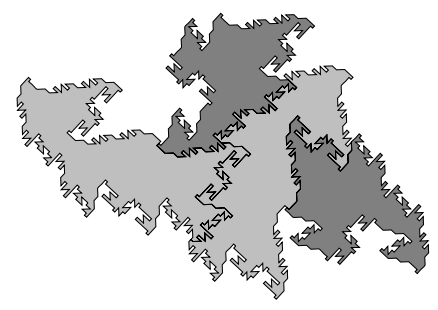

$$
T_{2}^{*}=L_{\theta}^{-1}\left(\left(\bigcup_{i=1}^{2} \gamma_{2, i}^{1}\left(T_{1}^{*}\right)\right) \cup\left(\bigcup_{i=1}^{3} \gamma_{2, i}^{2}\left(T_{2}^{*}\right)\right)\right) .
$$

In Figs. 10 and 11 the descomposition of each tile can be seen. Finally Fig. 12 shows a patch of the complete tiling. 
Fig. 12 Tiling

$\left\{\gamma\left(T_{1}^{*}\right), \gamma\left(T_{2}^{*}\right): \gamma \in \Gamma\right\}$

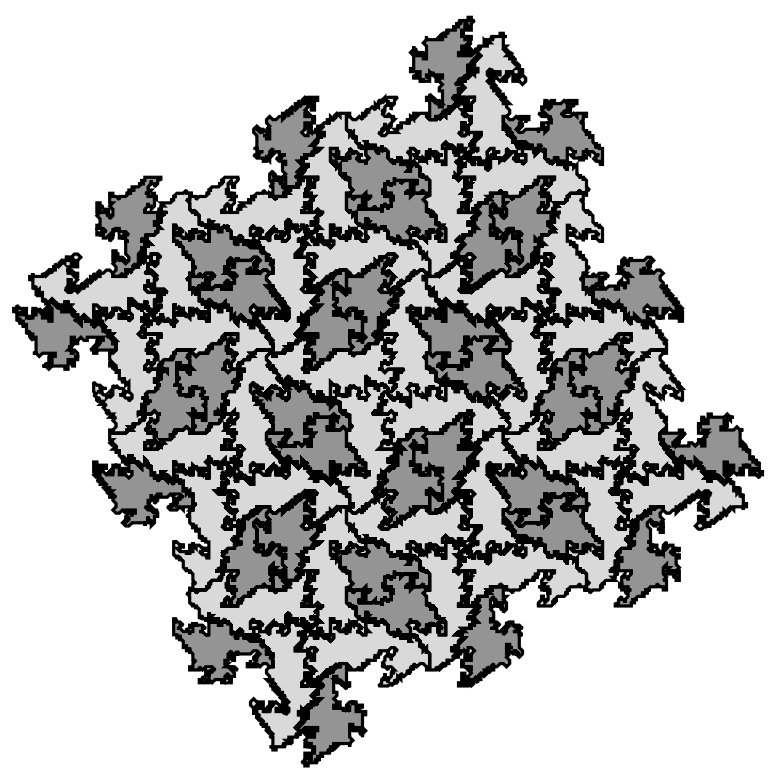

Acknowledgements The author gratefully acknowledges many helpful suggestions of Ursula Molter and Michael Lacey during the preparation of the paper.

\section{References}

1. Bedford, T.: Dimension and dynamics for fractal recurrent sets. J. Lond. Math. Soc. 33(2), 89-100 (1986)

2. Dekking, F.M.: Recurrent sets. Adv. Math. 44, 78-104 (1982)

3. Dekking, F.M.: Replicating superfigures and endomorphisms of free groups. J. Comb. Theory Ser. A 32, 315-320 (1982)

4. Duvall, P., Keesling, J., Vince, A.: The Hausdorff dimension of the boundary of a self-similar tile. J. Lond. Math. Soc. 61, 649-760 (2000)

5. Flaherty, T., Wang, Y.: Haar-type multiwavelet bases and self-affine multi-tiles. Asian J. Math. 3(2), 387-400 (1999)

6. González, A.L., Moure, M.C.: Haar bases and wavelet sets for crystallographic groups. Comunicación en la Conferencia Internacional de Análisis Armónico y Aplicaciones, Merlo, Arg. (2006)

7. Gröchenig, K., Madych, W.R.: Multiresolution analysis, Haar bases, and self-similar sets. IEEE Trans. Inf. Theory 38, 556-568 (1994)

8. Grünbaum, B., Shephard, G.S.: Tilings and Patterns. Freeman, New York (1987)

9. Guo, K., Labate, D., Lim, W., Weiss, G., Wilson, E.: Wavelets with composite dilations and their MRA properties. Appl. Comput. Harmon. Anal. 20(2), 202-236 (2006)

10. Ito, S.: On the fractal curves induced from the complex radix expansion. Tokyo J. Math. 12(2), 299320 (1989)

11. Kenyon, R., Li, J., Strichartz, R., Wang, Y.: Geometry of self-affine tiles II. Indiana J. Math. 48(1), 24-42 (1999)

12. Moure, M.C., Panzone, P.: Graph directed reptiles of $\mathbb{R}^{2}$. Ric. Mat. LIII(2), 213-229 (2004)

13. Moure, M.C.: Generación y análisis de conjuntos autoafines y multi-autoafines que embaldosan el plano. Tesis Doctoral, Univ. Nac. del Sur, Bahía Blanca, Arg. (2004)

14. Strichartz, R., Wang, Y.: Geometry of self-affine tiles I. Indiana J. Math. 48(1), 1-23 (1999)

15. Vince, A.: Self-replicating tiles and their boundary. Discrete Comput. Geom. 21, 463-476 (1999) 